\title{
DETERMINING THE INCLINATION OF THE ROTATION AXIS OF A SUN-LIKE STAR
}

\author{
L. GIZON ${ }^{1,2}$ AND S. K. SolanKI ${ }^{2}$ \\ Received 2002 December 3; accepted 2003 February 7
}

\begin{abstract}
Asteroseismology provides us with the possibility of determining the angle, $i$, between the direction of the rotation axis of a pulsating Sun-like star and the line of sight. A knowledge of $i$ is important not just for obtaining improved stellar parameters, but also in order to determine the true masses of extrasolar planets detected from the radial velocity shifts of their central stars. By means of Monte Carlo simulations, we estimate the precision of the measurement of $i$ and other stellar parameters. We find that the inclination angle can be retrieved accurately when $i \gtrsim 30^{\circ}$ for stars that rotate at least twice as fast as the Sun.
\end{abstract}

Subject headings: planetary systems — stars: fundamental parameters — stars: oscillations — stars: rotation

\section{INTRODUCTION}

For an Earth-based observer the rotation axis of the Sun is almost perpendicular to the line of sight. Traditionally, the solar rotation axis has been approximated to be exactly perpendicular to the ecliptic plane for helioseismic investigations of spatially unresolved oscillation data. An exception concerns the search for oblique rotation of the Sun's core (Goode \& Thompson 1992; Gough, Kosovichev, \& Toutain 1995). The rotation axes of stars are, however, randomly distributed in space. Since the visibility of the pulsation modes with various azimuthal orders $m$ is a function of the angle between the rotation axis and the line of sight, $i$, this angle cannot be ignored in asteroseismology. The presence of random $i$-values not only affects the method to measure oscillation mode parameters, but asteroseismology conversely provides us with the possibility of determining $i$, a parameter that in general is very poorly determined. Space missions such as COROT of CNES (Baglin et al. 2001) and Eddington of ESA (Favata, Roxburgh, \& ChristensenDalsgaard 2000) are expected to deliver the data necessary to do high-precision asteroseismology on a large number of stars.

The surface rotation rate of a star is one of its fundamental parameters and has been well studied. The standard method of deducing the rotation rate is to consider the widths of spectral lines. This technique only gives $v \sin i$, however, where $v$ is the equatorial rotation velocity at the stellar surface. Asteroseismology can in principle provide measurements of the angular velocity $\Omega$ and the inclination angle $i$. From these three measurements it is possible to determine the stellar radius, another fundamental parameter, without knowledge of stellar structure and evolution.

Knowledge of $i$ is important not just for obtaining improved stellar parameters, but also in order to determine the masses of extrasolar planets. The standard technique used to detect such planets is to look for periodic Doppler shifts in the spectrum of the central star of the extrasolar planetary system (Mayor \& Queloz 1995; Noyes et al. 1997; Marcy \& Butler 2000). This technique, however, only

${ }^{1}$ W. W. Hansen Experimental Physics Laboratory, Stanford University, Stanford, CA 94305.

2 Max-Planck-Institut für Aeronomie, Postfach 20, Katlenburg-Lindau D-37191, Germany. returns $M_{p} \sin i_{p}$, where $M_{p}$ is the mass of the orbiting body and $i_{p}$ is the inclination of the normal to its orbital plane relative to the line of sight. Clearly, the mass estimate obtained in this manner is a lower limit. Since $i$ and $i_{p}$ are expected to be similar (see below), a knowledge of $i$ would help to improve the mass estimates of extrasolar planets considerably and would distinguish also misidentified brown dwarfs in orbits with small $i_{p}$ from bona fide planets. In the solar system $i$ and $i_{p}$ differ by less than $10^{\circ}$ for all the planets excluding Pluto. In addition, currently favored theories of planetary system formation predict that the orbital plane of planets should nearly coincide with the equatorial plane of the central star (Safronov 1972; Lissauer 1993). An alternative technique for detecting planets involves looking for planetary transits in photometric data. So far this technique has uncovered only a couple of such systems (Charbonneau et al. 2000; Henry et al. 2000; Udalski et al. 2002; Konacki et al. 2003; Dreizler et al. 2003), compared to a total of over 100 planets detected using radial velocities. However, missions such as COROT, Eddington, and Kepler aim at discovering many such systems. Since for transiting planets $i_{p}$ is known to high accuracy (Brown et al. 2001), a comparison with the independently measured $i$ of the central stars would allow a direct test of the theoretical prediction that $i_{p}$ and $i$ are very similar. Clearly, there are many reasons to attempt to measure $i$.

Here we present a technique employing low-degree nonradial oscillations to determine $i$ for sufficiently rapidly rotating stars. The technique makes use of the fact that the ratio of amplitudes of the $m= \pm 1$ and 0 components of dipole oscillations is a strong function of $i$. Similarly, the amplitudes of the peaks in quadrupole multiplets exhibit different dependences on $i$. This technique is thus similar to using the ratios of $\sigma\left(\Delta M_{J}= \pm 1\right)$ to $\pi\left(\Delta M_{J}=0\right)$ components of Zeeman split atomic transitions to determine the angle of the magnetic field vector relative to the line of sight, a standard procedure in Zeeman magnetometry. By studying solar dipole modes of oscillation, Gough et al. (1995) were able to measure the inclination of the Sun's rotation axis within $5^{\circ}$ of the true value.

This technique also provides $\Omega$, the angular velocity. For active Sun-like stars $\Omega$ can also be determined by following surface tracers. By comparing the $\Omega$ determined from oscillations with the angular velocity from tracers, inferences can be made on the stellar differential rotation. A 
knowledge of this quantity, particularly for more rapidly rotating stars, would be of great interest (e.g., for dynamo theories). Alternatively, for those stars for which accurate astrometrically determined parallaxes are known and from which radii $R_{*}$ have been determined, a comparison of $\Omega R_{*} \sin i$ obtained from asteroseismology with $v \sin i$ determined spectroscopically could set limits on the differential rotation. We note that differential rotation could be determined seismically by measuring the rotational splitting frequencies of the quadrupole modes with $m= \pm 1$ and \pm 2 .

In this paper we simulate a large number of realizations of oscillation power spectra seen in intensity with known values of the stellar rotation and of the inclination angle. We then fit a parametric model to each power spectrum with a maximum likelihood technique to estimate $i, \Omega$, and other mode parameters. The distribution of the measured values of $i$ indicates how precise a measurement can be. In order to assess the feasibility of the technique, we assume that only a single multiplet, $l=1$ or 2 , is observed, whereas in reality we expect a number of multiplets to be accessible. In practice, the information from tens of modes should be combined to better constrain $i$. Although we are investigating a problem that has not been studied before, we employ many results from helioseismology. We choose to give a fairly detailed description in order to state underlying assumptions and to reach out to a relatively broad audience.

\section{EFFECT OF ROTATION ON STELLAR OSCILLATIONS}

Stars like the Sun undergo global acoustic oscillations driven by near-surface turbulent convection. The pulsation frequencies $\omega_{n l}$ of eigenmodes with radial order $n$ and spherical harmonic degree $l$ are characteristic of the spherically symmetric structure of a star (Brown \& Gilliland 1994). For distant Sun-like stars, observations are mostly sensitive to high-order acoustic modes with $l \leq 2$, i.e., radial, dipole, and quadrupole $p$-modes. Because low-degree frequencies satisfy a relatively simple asymptotic relation (Tassoul 1980) in which the large separation $\omega_{n l}-\omega_{n-1, l}$ and the small separation $\omega_{n 0}-\omega_{n-1,2}$ depend weakly on $n$, the degree $l$ of a multiplet can in principle be identified without ambiguity in the oscillation power spectrum (Fossat 1981). A solar oscillation power spectrum for 200 days of observation of the total irradiance (Fröhlich et al. 1997) is shown in Figure 1. Many attempts have been made to detect $p$-modes on other Sun-like stars. So far they have only been clearly detected on $\alpha$ Cen A (Bouchy \& Carrier 2001; Schou \& Buzasi 2001; Bedding et al. 2002).

Rotation removes the $(2 l+1)$-fold degeneracy of the frequency of oscillation of the mode $(n, l)$. The nonradial modes of a rotating star are thus labeled with a third index, the azimuthal order $m$, which takes integer values from $-l$ to $+l$. When the angular velocity of the star, $\Omega$, is small, the effect of rotation on mode frequencies can be treated as a small perturbation. In the case of rigid-body rotation, and to a first order of approximation, the frequency of the mode $(n, l, m)$ is given by (Ledoux 1951)

$$
\omega_{n l m}=\omega_{n l}+m \Omega\left(1-C_{n l}\right) .
$$

The kinematic splitting, $m \Omega$, is corrected for the effect of the Coriolis force through the dimensionless quantity $C_{n l}>0$, whose value depends on the oscillation eigenfunctions of the nonrotating star. High-order, low-degree solar oscillations have $C_{n l}<10^{-2}$; rotational splitting is dominated by advection. We note that the rotation-induced frequency shift would not be linear in $m$ if the angular velocity $\Omega$ were to vary with latitude (e.g., Hansen, Cox, \& Van Horn 1977).

To the next order of approximation, centrifugal forces distort the equilibrium structure of the star. This results in an additional frequency perturbation (independent of the sign of $m$ ) that scales like the small parameter

$$
\frac{\Omega^{2} R^{3}}{\mathscr{G} M}
$$

i.e., the ratio of the centrifugal to the gravitational forces at the stellar surface (Saio 1981; Gough \& Thompson 1990). Here $R$ denotes the radius of the star, $M$ its mass, and $\mathscr{G}$ the universal constant of gravity. Second-order rotational effects are negligible in the Sun (Dziembowski \& Goode 1992). These effects are, however, significant for faster rotating Sun-like stars (Kjeldsen et al. 1998). Other perturbations, such as a large-scale magnetic field, may introduce further corrections to the pulsation frequencies (Gough \& Thompson 1990).

In this paper we only consider first-order rigid rotation and substitute $\omega_{n l}+m \Omega$ for $\omega_{n l m}$. Our purpose is to assess the feasibility of measuring the basic rotation parameters $\Omega$ and $i$. In an inertial frame $\mathscr{R}^{\prime}$ with polar axis coincident with the angular velocity vector $\Omega$, scalar eigenfunctions are proportional to a spherical harmonic function $Y_{l}^{m}\left(\theta^{\prime}, \phi^{\prime}\right)$, where $\theta^{\prime}$ and $\phi^{\prime}$ are the colatitude and longitude defined in the spherical-polar coordinate system associated with $\mathscr{R}^{\prime}$.

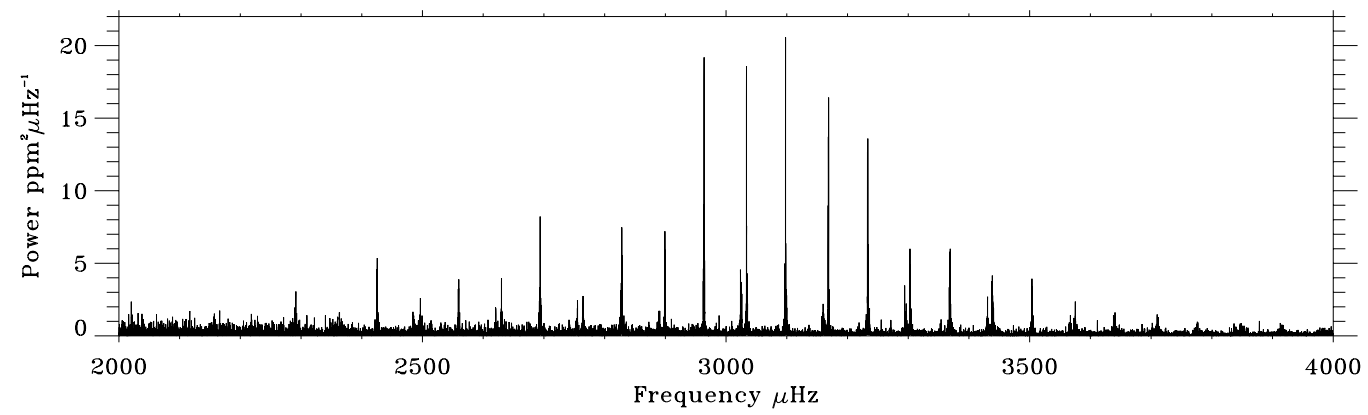

FIG. 1.- Solar oscillation power spectrum for 200 days of observation of the total irradiance (Fröhlich et al. 1997). The data are from the VIRGO experiment aboard the ESA/NASA Solar and Heliospheric Observatory $(\mathrm{SOHO})$. The global modes of oscillation are ordered in sequence: $(n-1, l=2),(n, l=0)$, and $(n, l=1)$ with radial order $n$ increasing with frequency. The large frequency separation is $\left(\omega_{n, l}-\omega_{n-1, l}\right) / 2 \pi \sim 135 \mu \mathrm{Hz}$, and the small separation is $\left(\omega_{n, l=0}-\omega_{n-1, l=2}\right) / 2 \pi \sim 10 \mu \mathrm{Hz}$. 
Under the approximation that the intensity fluctuation due to a mode of oscillation is proportional to a scalar eigenfunction measured at the stellar surface (such as the Lagrangian pressure perturbation), the brightness variations due to the free oscillations of a star may be written as a linear combination of eigenmodes:

$$
I^{\prime}\left(t, \theta^{\prime}, \phi^{\prime}\right)=\Re \sum_{n l} \sum_{m=-l}^{l} A_{n l m} Y_{l}^{m}\left(\theta^{\prime}, \phi^{\prime}\right) e^{\mathrm{i} \omega_{n l m} t},
$$

where $A_{n l m}$ are complex amplitudes, $t$ denotes time, and $\mathfrak{R}$ takes the real part of the expression. A more accurate expression for $I^{\prime}$ requires an explicit relationship between mode displacement and light-flux perturbation (e.g., Toutain \& Gouttebroze 1993).

To obtain the intensity that an Earth-based observer would measure, it is convenient to transform to an inertial frame $\mathscr{R}$ with polar axis pointing toward the observer, inclined by the angle $i$ with respect to $\boldsymbol{\Omega}$. Colatitude $\theta$ and longitude $\phi$ are spherical-polar coordinates defined in $\mathscr{R}$. For an appropriate choice of longitude origins, spherical harmonics expressed in $\mathscr{R}^{\prime}$ and $\mathscr{R}$ are related linearly according to (Messiah 1959)

$$
Y_{l}^{m}\left(\theta^{\prime}, \phi^{\prime}\right)=\sum_{m^{\prime}=-l}^{l} Y_{l}^{m^{\prime}}(\theta, \phi) r_{m^{\prime} m}^{(l)}(i),
$$

where the rotation matrix $\boldsymbol{r}^{(l)}$ is real and unitary.

According to Wigner's formula (see Messiah 1959), each rotation matrix element can be written explicitly as a homogeneous polynomial of total degree $2 l$ in the two variables $\sin (i / 2)$ and $\cos (i / 2)$. Inserting equation (4) into equation (3), we obtain intensity variations expressed in the frame with polar axis $(\theta=0)$ pointing toward the observer:

$$
I(t, \theta, \phi)=\Re \sum_{n l m m^{\prime}} A_{n l m} Y_{l}^{m^{\prime}}(\theta, \phi) r_{m^{\prime} m}^{(l)}(i) e^{\mathrm{i} \omega_{n l m} t} .
$$

The spherical harmonic projection $\left(l, m^{\prime}\right)$ is given by a linear combination of eigenmodes $(l, m)$. From the above equation, we derive the observed disk-integrated intensity signal, $I(t)$ :

$$
I(t)=\int_{0}^{2 \pi} d \phi \int_{0}^{\pi / 2} d \theta I(t ; \theta, \phi) W(\theta) \cos \theta \sin \theta,
$$

where $W(\theta)$ is the limb-darkening function. Because the function $Y_{l}^{m^{\prime}}(\theta, \phi)$ is proportional to $\exp \left(\mathrm{i} m^{\prime} \phi\right)$, components with $m^{\prime} \neq 0$ vanish upon integration over azimuth, and

$$
I(t)=\Re \sum_{n l m} V_{l} A_{n l m} r_{0 m}^{(l)}(i) e^{\mathrm{i} \omega_{n l m} t},
$$

with the visibility factor $V_{l}$ given by

$$
V_{l}=2 \pi \int_{0}^{\pi / 2} Y_{l}^{0}(\theta) W(\theta) \cos \theta \sin \theta d \theta .
$$

For each $(l, n)$ there are $2 l+1$ visible peaks in the power spectrum, as is expected for a steady perturbation such as rotation. The quantity $V_{l}^{2}$ is an estimate of the geometrical visibility of the total power in a multiplet $(l, n)$ as a function of $l$. The solar limb-darkening function quoted by Pierce (2000) implies $\left(V_{1} / V_{0}\right)^{2}=0.50$ and $\left(V_{2} / V_{0}\right)^{2}=0.17$. These estimates are crude (see Toutain \& Gouttebroze 1993). However, the ratios $V_{l} / V_{0}$ are unimportant to the present study as we are interested in the relative power between azimuthal modes with common values of $l$ and $n$.

Assuming that there is energy equipartition between modes with different azimuthal order, we write amplitudes $A_{n l m}$ in the form

$$
A_{n l m}=\left|A_{n l}\right| e^{\mathrm{i} \phi_{n l m}},
$$

where the magnitude $\left|A_{n l}\right|$ is independent of $m$ and $\phi_{n l m}$ is an arbitrary phase. Using this assumption, consistent with the solar data, together with equation (7), we find that the dependence of mode power on azimuthal order $m$ is given by

$$
\mathscr{E}_{\operatorname{lm}}(i)=\left[r_{0 m}^{(l)}(i)\right]^{2}
$$

Matrix elements $r_{0 m}^{(l)}(i)$ are explicitly given by Messiah (1959) in terms of associated Legendre functions, $P_{l}^{m}$ :

$$
\mathscr{E}_{l m}(i)=\frac{(l-|m|) !}{(l+|m|) !}\left[P_{l}^{|m|}(\cos i)\right]^{2} .
$$

The above equation links mode visibility to inclination angle $i$ (see also Dziembowski 1977; Toutain \& Gouttebroze 1993). It provides the basic information required to later extract $i$ from photometric measurements. It is, however, unknown whether the key assumption, equation (9), remains valid for very fast rotators as rotation affects convection and therefore the mechanism by which acoustic modes are excited. For dipole multiplets, $l=1$, the observed mode power (eq. [11]) is given by

$$
\begin{aligned}
\mathscr{E}_{1,0}(i) & =\cos ^{2} i, \\
\mathscr{E}_{1, \pm 1}(i) & =\frac{1}{2} \sin ^{2} i
\end{aligned}
$$

For quadrupole multiplets, $l=2$, we have

$$
\begin{aligned}
\mathscr{E}_{2,0}(i) & =\frac{1}{4}\left(3 \cos ^{2} i-1\right)^{2}, \\
\mathscr{E}_{2, \pm 1}(i) & =\frac{3}{8} \sin ^{2}(2 i), \\
\mathscr{E}_{2, \pm 2}(i) & =\frac{3}{8} \sin ^{4} i .
\end{aligned}
$$

It is worth noting the symmetries $\mathscr{E}_{l m}(-i)=\mathscr{E}_{l m}(\pi-i)=$ $\mathscr{E}_{l m}(i)$. Knowledge of $\mathscr{E}_{l m}(i)$ is not enough to fully specify the direction and sense of the rotation axis, but only $|i| \bmod \pi$. When the rotation axis is aligned with the line of sight $(i=0$ $\bmod \pi$ ), only the mode $m=0$ is visible and rotation cannot be inferred. Notice also that $\sum_{m} \mathscr{E}_{l m}(i)=1$, so that $\mathscr{E}_{l m}$ represents the relative power in the mode $m$ within a multiplet $(n, l)$.

\section{MODELING OSCILLATION POWER SPECTRA}

In the previous section we studied the intensity variations due to the free oscillations of a star with an arbitrary orientation of the rotation axis. We found that the brightness variations can be approximated by

$$
I(t)=\sum_{m=-l}^{l} \sqrt{\mathscr{E} l m(i)} \cos \left[\left(\omega_{n l}+m \Omega\right) t+\phi_{m}\right],
$$

when considering only the contribution from a single multiplet $(n, l)$. The observed power in the azimuthal component $m$ is given by $\mathscr{E}_{l m}(i)$, and $\phi_{m}$ is an arbitrary phase. In Sun-like 
stars, oscillations are, however, excited by near-surface turbulent convection. The above model is too simple as it ignores the stochastic nature of stellar pulsations (Woodard 1984). Oscillations also have a finite lifetime determined by their interaction with convection. In this section we give a more realistic description of the statistical properties of the oscillation signal in Fourier space.

The observed brightness variations of a star are presumed to be given by the function $I(t)$ recorded over a large observation time interval of length $T$, at a sufficiently high cadence (say, less than 1 minute). Since pulsations are forced by turbulence, the signal is a random sample drawn from some probability distribution. Neglecting edge effects introduced by the time window, we assume that $I(t)$ is a stationary process. We denote by $I\left(\omega_{j}\right)$ the fast Fourier transform of $I(t)$ sampled at the angular frequency $\omega_{j}=2 \pi j / T$. A random variable is fully specified by its expectation value, $E$, and higher order moments (in the sense of ensemble averages). Here $I\left(\omega_{j}\right)$ is complex with zero mean, $E\left[I\left(\omega_{j}\right)\right]=0$, and stationarity implies that frequency bins are uncorrelated:

$$
E\left[\overline{I\left(\omega_{j}\right)} I\left(\omega_{j^{\prime}}\right)\right]=0 \text { for } j \neq j^{\prime},
$$

where the bar denotes the complex conjugate. Foglizzo et al. (1998) showed that low-degree modes are essentially uncorrelated. This is a consequence of the fact that there is a very large number of excitation events per damping time. The central limit theorem ensures that mode amplitudes converge to independent Gaussian distributions. The signal $I\left(\omega_{i}\right)$ can thus be modeled by a sum of independent complex Gaussian random variables:

$$
I\left(\omega_{j}\right)=\sum_{m} \sigma_{m}\left(\omega_{j}\right) \mathscr{N}_{m, j}+\sigma^{\mathrm{n}} \cdot \mathscr{N}_{j}^{\mathrm{n}} .
$$

The symbol $\mathscr{N}$ denotes a complex Gaussian random variable with zero mean and unit variance, $E\left[|\mathscr{N}|^{2}\right]=1$. The standard deviation of a mode amplitude, denoted by the function $\sigma_{m}(\omega)$, is large for $\omega$ near the resonant frequency $\omega_{n l}+m \Omega$ (see below). The distributions $\mathscr{N}_{m, j}$ are all independent of each other. The additional term $\sigma^{\mathrm{n}} \mathcal{N}_{j}^{\mathrm{n}}$ denotes uncorrelated Gaussian noise with standard deviation $\sigma^{\mathrm{n}}$. The origin of this noise is both stellar (convection) and instrumental (e.g., photon noise). For simplicity, the noise level, $\sigma^{\mathrm{n}}$, is assumed to be frequency independent over a small frequency interval around $\omega_{n l}$.

In order to obtain $\sigma_{m}(\omega)$, one should in principle solve the inhomogeneous wave equations once a model for wave damping and excitation has been specified. Here, however, we parameterize the variance $\sigma_{m}^{2}$ in the form

$$
\sigma_{m}^{2}(\omega)=S \mathscr{E}_{l m}(i) L_{n l}(\omega-m \Omega) .
$$

The constant $S$ gives the overall amplitude of the power, and the weights $\mathscr{E}_{l m}(i)$ give the $m$-dependent visibility as a function of inclination angle $i$ (see $\S 2$ ). The line shape, $L_{n l}(\omega)$, is a real positive function that becomes large for $\omega$ near the resonant frequency $\omega_{n l}$. We choose the standard Lorentzian line profile (e.g., Anderson, Duvall, \& Jefferies 1990) appropriate for describing an exponentially damped oscillator:

$$
L_{n l}(\omega)=\left[1+\left(\frac{\omega-\omega_{n l}}{\Gamma / 2}\right)^{2}\right]^{-1},
$$

where the damping rate $\Gamma$ represents the FWHM of $L_{n l}(\omega)$. Notice that equation (21) only gives the positive-frequency part of the spectrum; the negative-frequency part does not contain extra information and can be deduced from the relation $I(-\omega)=\overline{I(\omega)}$.

Since the sum of independent Gaussian random variables is a Gaussian variable, the Fourier spectrum (eq. [19]) at frequency $\omega_{j}$ can be written in terms of a single complex normal distribution, $\mathscr{N}_{j}$ :

$$
I\left(\omega_{j}\right)=\left[S \sum_{m} \mathscr{E}_{l m}(i) L_{n l}\left(\omega_{j}-m \Omega\right)+N\right]^{1 / 2} \mathscr{N}_{j} .
$$

We introduced the notation $N=\left(\sigma^{\mathrm{n}}\right)^{2}$. The traditional method to generate a complex Gaussian distribution is called the Box-Muller method. Given a uniform distribution on $[0,1], U_{j}$, and a uniform distribution on $[0,2 \pi], \Theta_{j}$, the random variable

$$
\mathscr{N}_{j}=\sqrt{-\ln U_{j}} e^{\mathrm{i} \Theta_{j}}
$$

is complex Gaussian with independent real and imaginary parts and unit variance. From equations (22) and (23), we see that a realization of the power spectrum is given by

$$
P\left(\omega_{j}\right)=\left|I\left(\omega_{j}\right)\right|^{2}=-\ln \left(U_{j}\right) \mathscr{P}\left(\omega_{j}\right),
$$

where $\mathscr{P}$ is the expectation value of the power spectrum,

$$
\mathscr{P}\left(\omega_{j}\right)=S \sum_{m} \mathscr{E}_{l m}(i) L_{n l}\left(\omega_{j}-m \Omega\right)+N .
$$

We now have an expression for generating realizations of a stellar oscillation power spectrum. Because $L_{n l}\left(\omega_{n l}\right)=1$, it makes sense to refer to $S / N$ as the signal-to-noise ratio in the power spectrum. Since $-\ln \left(U_{j}\right)$ is an exponential distribution with unit mean and variance, the probability density function of the random variable $P\left(\omega_{j}\right)$ is given by

$$
f\left(P_{j}\right)=\frac{1}{\mathscr{P}\left(\omega_{j}\right)} \exp \left[-\frac{P_{j}}{\mathscr{P}\left(\omega_{j}\right)}\right],
$$

where $f\left(P_{j}\right)$ describes the probability that $P\left(\omega_{j}\right)$ takes a particular value $P_{j}$ (Woodard 1984; Duvall \& Harvey 1986).

Figure 2 shows plots of the expectation value of the power spectrum, $\mathscr{P}(\omega)$, for various values of the inclination angle $i$. The left-hand panels in Figure 2 are for dipole multiplets $l=1$, and the right-hand panels for quadrupole multiplets $l=2$. In these plots the parameters are $\Gamma=\Gamma_{\odot}$ and $\Omega=6 \Omega_{\odot}$, where $\Gamma_{\odot} / 2 \pi=1 \mu \mathrm{Hz}$ and $\Omega_{\odot} / 2 \pi=0.5 \mu \mathrm{Hz}$ are characteristic solar values for the line width and the angular velocity. For noiseless data, the dependence of the power at different frequencies on $i$ is clearly evident, and it is possible to distinguish between different $i$-values relatively easily.

To illustrate the effect of stochastic excitation, Figure 3 shows two realizations, $P(\omega)$, of an $l=2$ power spectrum for $i=30^{\circ}$ and $80^{\circ}$, together with the expectation values denoted by the thick curves. A solar-like background noise was prescribed $(S / N=100)$. Although realization noise is important, the two spectra can be distinguished from each other.

\section{ESTIMATION OF STELLAR PARAMETERS}

In the previous section we described a simple statistical model for the stellar oscillation power spectrum. This model 

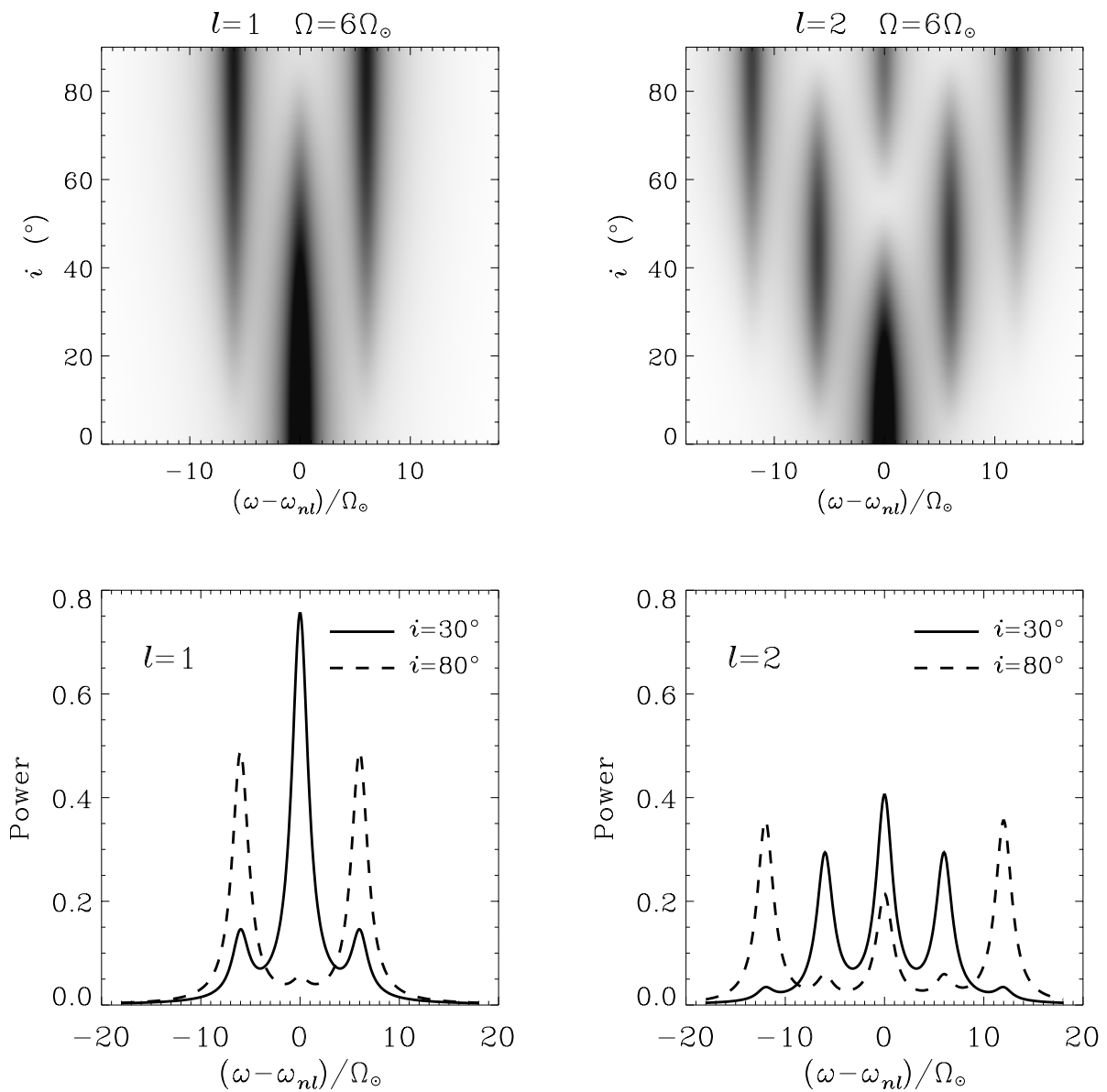

FIG. 2.-Expectation value of the power spectrum, $\mathscr{P}(\omega)$, for dipole and quadrupole multiplets as a function of the inclination angle $i$. The left-hand panels are for dipole multiplets, $l=1$, and the right-hand panels are for quadrupole multiplets, $l=2$. The parameters are $\Gamma=\Gamma_{\odot}$ and $\Omega=6 \Omega_{\odot}$, where $\Gamma_{\odot} / 2 \pi=$ $1 \mu \mathrm{Hz}$ and $\Omega_{\odot} / 2 \pi=0.5 \mu \mathrm{Hz}$ are characteristic solar values for the line width and the angular velocity. The bottom panels show the power for the specific values $i=30^{\circ}$ (solid lines) and $80^{\circ}$ (dashed lines). There is no background noise in these plots.
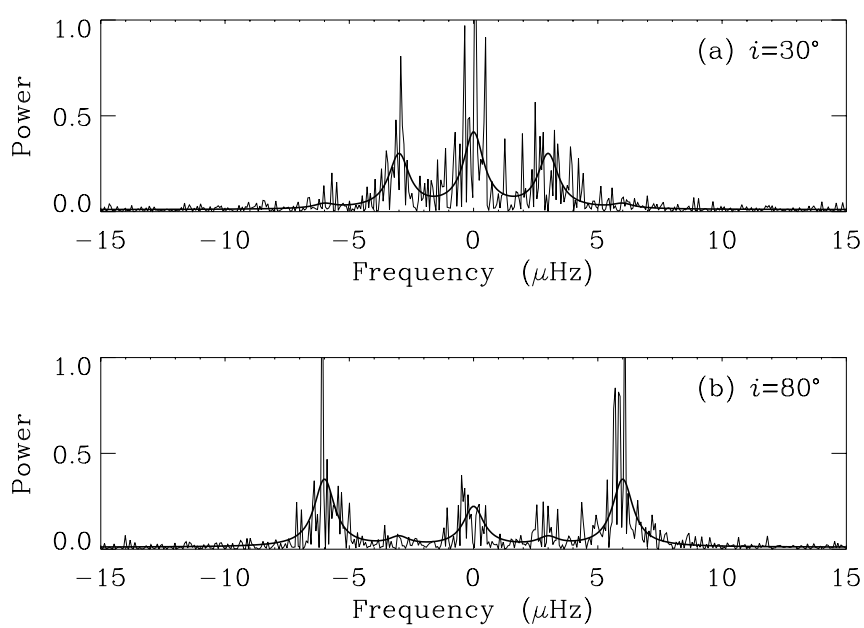

FIG. 3.-Two realizations of the power spectrum of an $l=2$ multiplet vs. centered frequency $\left(\omega-\omega_{n l}\right) / 2 \pi$. The stellar rotation is $\Omega=6 \Omega_{\odot}$, and the mode line width is $\Gamma=\Gamma_{\odot} .($ a $)$ corresponds to an inclination angle $i=30^{\circ}$, and $(b)$ is for $i=80^{\circ}$. A signal-to-noise ratio $S / N=100$ has been prescribed, and the simulation corresponds to 6 months of uninterrupted observations. Expectation values of the power are overplotted. depends on a minimal set of physical parameters $\left(\omega_{n l}, \Gamma, \Omega\right.$, i) and the overall signal and noise levels $(S, N)$. In this section we describe an algorithm that allows these parameters to be estimated from a realization of the power spectrum. We use the maximum likelihood method, which is commonly used in helioseismology (e.g., Anderson et al. 1990; Schou 1992; Toutain \& Appourchaux 1994; Appourchaux, Gizon, \& Rabello-Soares 1998; Appourchaux et al. 2000).

We consider a section of the spectrum that includes the $2 l+1$ peaks of a given multiplet $(l, n)$. The spherical harmonic degree $l$ is either 1 or 2 . We denote by $\lambda$ the set of parameters that we want to estimate:

$$
\lambda=\left\{\lambda_{k}\right\}\left\{i, \Omega, \omega_{n l}, \Gamma, S, N\right\} .
$$

Maximum likelihood estimators involve specifying the joint probability density function for the sample data $\left\{P_{j}\right\}$. For a given frequency $\omega_{j}$, the probability that the power takes the particular value $P_{j}$ is given by the probability density function, $f\left(P_{j}\right)$ (see eq. [26]). We write $f\left(P_{j} \mid \lambda\right)$ to indicate the dependence on the parameters $\lambda$. Because frequency bins are independent, the joint probability density function is simply the product of $f\left(P_{j} \mid \lambda\right)$ for the index $j$ spanning the frequency interval of interest. The likelihood function $F(\lambda)$ is another name for the joint probability function evaluated 
at the sample data

$$
F(\lambda)=\prod_{j} f\left(P_{j} \mid \lambda\right) .
$$

The basic idea of maximum likelihood estimation is to choose estimates $\lambda^{*}$ so as to maximize the likelihood function. In practice, one minimizes

$$
\mathscr{L}(\lambda)=-\ln F(\lambda) .
$$

This gives the same result since the logarithm is a monotonic increasing function. The probability of observing the sample values is greatest if the unknown parameters are equal to their maximum likelihood estimates:

$$
\lambda^{*}=\arg \min _{\lambda}\{\mathscr{L}(\lambda)\} .
$$

In this paper we use the conjugate gradient method to find the parameters that minimize the function $\mathscr{L}$.

The method of maximum likelihood has many good properties (e.g., Kendall \& Stuart 1967; Rao 1973). The maximum likelihood estimate $\lambda^{*}$ is not biased as the sample size tends to infinity. Moreover, for large sample size, $\lambda^{*}$ will have an approximate multinormal distribution centered on the true parameter value $\lambda$. Maximum likelihood estimators are also minimum variance estimators. Furthermore, when the model is misspecified, $\lambda^{*}$ will still have a well-defined probability distribution and will be approximately normally distributed. In our case we have a finite sample size, since $T$ is limited to a few months. There is no guarantee that the maximum likelihood estimator will be normally distributed or even unbiased. Note also that the distribution of $i^{*}$ has to be periodic since $\mathscr{L}$ only depends on $|i| \bmod \pi$.

In order to derive the correct probability distributions of the likelihood estimates, we run Monte Carlo simulations (e.g., Toutain \& Appourchaux 1994). The method consists of simulating a large number of realizations of a power spectrum and then fitting each realization to construct the distributions of the measured parameters. Monte Carlo simulations enable us to determine the bias and the precision associated with the measurement of each parameter $\lambda_{k}$. Ideally we would want to run simulations for each relevant point in $\lambda$-space and for varying observation times $T$. Because Monte Carlo simulations are time consuming, we decide to keep $\Gamma$ and $S / N$ fixed to their solar values, varying only $\Omega$ and $i$. For all simulations, the observation time is $T=6$ months.

\section{RESULTS}

In Figure 4 we show the results for one set of Monte Carlo simulations. Plotted are in Figures $4 a$ and $4 c$ the inclination angle $i^{*}$ and in Figures $4 b$ and $4 d$ the angular velocity in solar units, $\Omega^{*} / \Omega_{\odot}$, returned by the fit versus the inclination angle, $i$, that entered the computation of each realization. For this set we simulate a single $l=1$ triplet and the rotation frequency is $\Omega=6 \Omega_{\odot}$. For each value of the inclination angle $i$ ranging from $0^{\circ}$ to $90^{\circ}$, we computed 2000 realizations of the power spectrum. The initial guesses in $\omega_{n l}^{*}, \Gamma^{*}$, $S^{*}$, and $N^{*}$ for the fits to the simulated spectra are randomly distributed in some interval around the true parameter values. The random initial guess in $i^{*}$ is uniformly distributed between $0^{\circ}$ and $90^{\circ}$, whatever the true inclination angle. For $\Omega^{*}$, we started with two different initial guesses. The guesses are indicated by the dashed lines in Figures $4 b$ (for the results shown in Figs. $4 a$ and $4 b$ ) and $4 d$ (for Figs. $4 c$ and $4 d$ ). The guess for $\Omega$ shown in Figure $4 d$ is not too dissimilar from an initial guess based on $v \sin i$ measurements.

We note that most $i^{*}$ values returned by the fits lie within $\pm 5^{\circ}$ of the true $i$. However, the distribution of $i^{*}$ is highly non-Gaussian as $i$ tends to either $0^{\circ}$ or $90^{\circ}$. The accuracy is lower for small $i$-values in particular if a wrong initial guess of $\Omega^{*}$ is made (Fig. $4 c$ ). In this case the fits tend to either $i^{*}=0^{\circ}$ or $90^{\circ}$ for $i \lesssim 10^{\circ}$. The inaccuracies in $\Omega^{*}$ are also largest for small $i$, and systematically too low values are returned if the initial guess is too low (Fig. 4d).

The reason for this behavior lies in the fact that only the $m=0$ component is visible at $i \approx 0$ (Fig. 2). Hence, the oscillation spectrum does not provide any means of distinguishing between a (rapidly) rotating star observed almost pole-on and a nonrotating (or very slowly rotating) star with arbitrary $i$-value. In this case the maximum likelihood fit returns the solution closer to the initial guess (compare Figs. $4 b$ and $4 d$ ).

In Figure 5 we plot the same as Figure 4, but for an $l=2$ mode. On the whole, the results look similar. At most inclination angles the accuracy in the measurements of $i^{*}$ and $\Omega^{*}$ is higher than for $l=1$. The major exception is $i$ in the range $20^{\circ}-40^{\circ}$. The fitting procedure cannot decide between $i^{*} \approx i, \Omega^{*} \approx \Omega$ and $i^{*} \approx 90^{\circ}, \Omega^{*} \approx \Omega / 2$. Figure 2 again reveals the cause of this uncertainty. For $i \approx 20^{\circ}-40^{\circ}$ only the $m=0$ and \pm 1 components have significant power. The solution with $\Omega^{*} \approx \Omega / 2$ is achieved if the $m= \pm 1$ components are misidentified as $\Delta m= \pm 2$. This is only possible if simultaneously $i^{*} \approx 90^{\circ}$ is assumed (see Fig. 2). Unsurprisingly, this wrong solution is more commonly obtained when the initial guess of $\Omega^{*}$ is closer to $\Omega / 2$ than to $\Omega$ (Fig. $5 d$ ). For $i \gtrsim 80^{\circ}$ again two solutions are obtained, the correct one and $\Omega^{*} \approx 2 \Omega$ combined with $i^{*} \approx 30^{\circ}$. In this case the fitting procedure misidentifies the $m= \pm 2$ peaks as $m= \pm 1$ peaks.

The most reliable result is obtained by fitting dipole and quadrupole modes simultaneously. Figure 6 shows likelihood estimates for three multiplets $l=0,1,2$ combined. The ambiguities at $i \approx 20^{\circ}-40^{\circ}$ and $i \gtrsim 80^{\circ}$ present in the fits to $l=2$ alone are removed, while the scatter in $i^{*}$ and $\Omega^{*}$ is considerably smaller than for fits to $l=1$ peaks alone. Only the ambiguity at $i \lesssim 10^{\circ}$ remains. "Medians" and "error bars" are plotted in Figure 6. By construction $\frac{2}{3}$ of the points lie between error bars. Because the distributions of $i^{*}$ and $\Omega^{*}$ are definitely not Gaussian, these values are only indicative; they are not sufficient to assess the measurement precision. Also plotted in Figures $6 c$ and $6 d$ are the fitted frequency of the $l=0$ mode, $\omega_{n 0}^{*}$, and the line width, $\Gamma^{*}$, common to all the modes. The measurement accuracy of these parameters appears to be independent of the inclination angle $i$. Indeed, rotation has no effect on the singlet $l=0$. Including an $l=0$ mode in the minimization procedure helps in turn to measure $\Omega^{*}$ and $i^{*}$ from the dipole and quadrupole modes by reducing the uncertainty on $\Gamma^{*}$.

So far we have only considered rapidly rotating stars with rotational splitting considerably larger than the line width. We now turn to the case $\Omega=2 \Omega_{\odot}$ and repeat the analysis described above for $\Omega=6 \Omega_{\odot}$. The distribution of $i^{*}$ and $\Omega^{*}$ obtained by fitting 750 realizations to $l=0,1,2$ combined is shown in Figure 7. As expected, the accuracy of the deduced $i^{*}$ and $\Omega^{*}$ values is considerably lower now than for the more rapidly rotating stars. The individual azimuthal components in a multiplet are not resolved since $\Omega=2 \Gamma$. For $i \gtrsim 45^{\circ}$ the errors are found to be around $\pm 10^{\circ}$ for $i^{*}$ and 

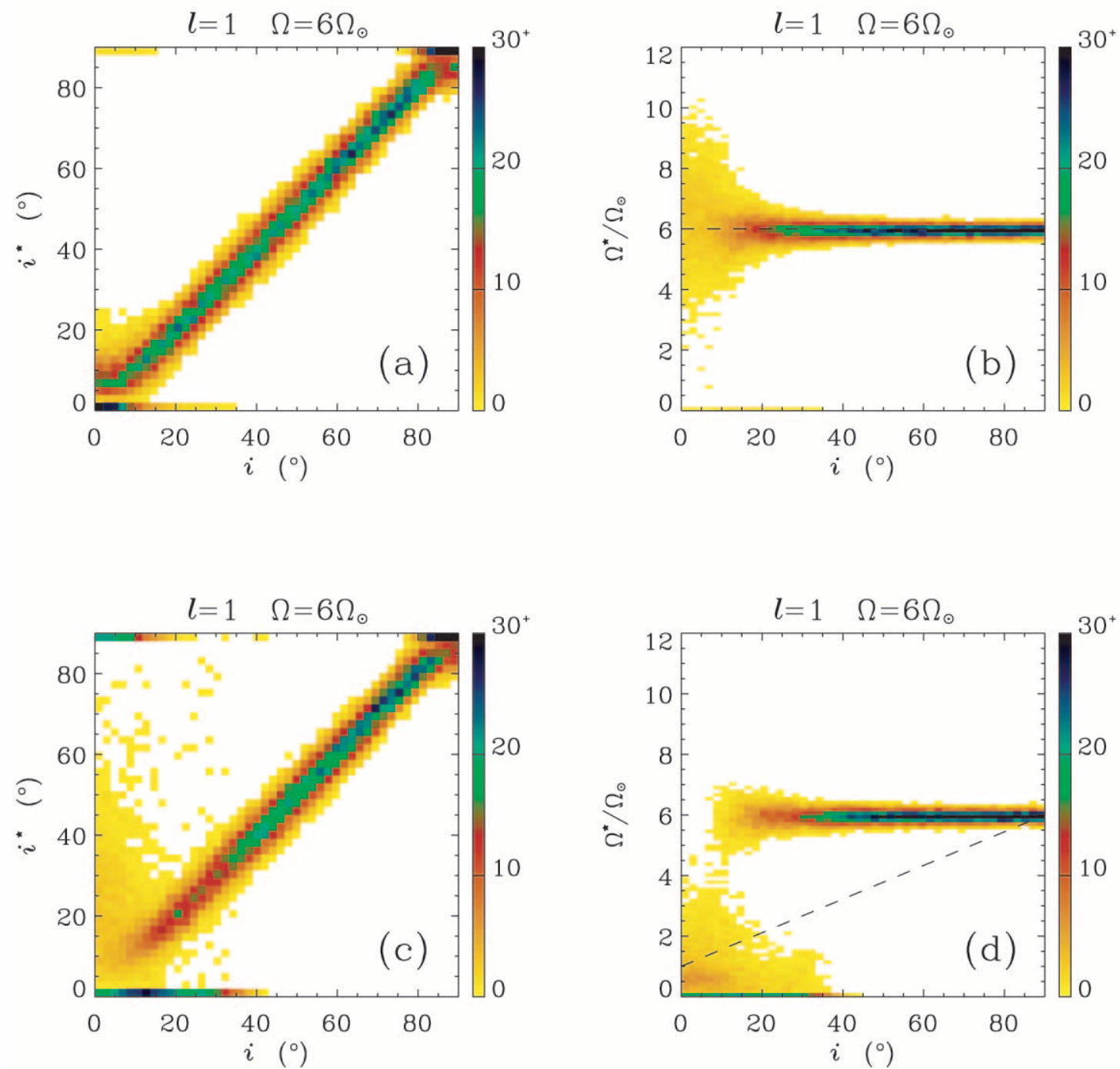

Fig. 4.-Maximum likelihood estimates $i^{*}$ and $\Omega^{*}$ deduced from an $l=1$ triplet vs. the true $i$. The true rotation frequency is $\Omega=6 \Omega \odot$. Plotted are the results of 2000 realizations. $(a)$ and $(b)$ differ from $(c)$ and $(d)$ in the initial guess for the rotation rate (indicated by the dashed lines). The scale indicates the percentage of the points falling into a bin. For $(a)$ and $(c)$ a bin is $2^{\circ}$. For $(b)$ and $(d)$ a bin is $0.12 \Omega_{\odot}$.

$5 \%-15 \%$ for $\Omega^{*} / \Omega$. At smaller $i$-values the fits tend to overestimate $i$ and the uncertainty for both $i^{*}$ and $\Omega^{*}$ becomes excessively large for decreasing $i$ but remains unchanged for $\omega_{n 0}^{*}$ and $\Gamma^{*}$. A comparison with Figure 7 reveals that the accuracy of these last two quantities is mostly independent of the rotation rate when $l=0,1,2$ are fitted together.

Although extremely useful, Monte Carlo simulations require long computations. A less reliable but straightforward method to obtain a formal error, $\sigma_{k}$, on the maximum likelihood estimate $\lambda_{k}^{*}$ is to expand $\mathscr{L}$ about the true parameter value $\lambda_{k}$. As mentioned earlier, in the limit of infinite sample size, $\lambda^{*}$ tends to a multinormal probability distribution that is asymptotically unbiased,

$$
E\left[\lambda_{k}^{*}\right]=\lambda_{k},
$$

and has minimum variance. An estimate of $\sigma_{k}$ is

$$
\sigma_{k}^{2}=\frac{1}{C_{k k}},
$$

where $C_{k k}$ is the $k$ th element on the diagonal of the inverse, $\boldsymbol{C}=\boldsymbol{H}^{-1}$, of the Hessian matrix given by

$$
H_{k k^{\prime}}=E\left[\frac{\partial^{2} \mathscr{L}}{\partial \lambda_{k} \partial \lambda_{k^{\prime}}}(\lambda)\right] .
$$

The formal error $\sigma_{k}$, called the Cramer-Rao lower bound, is a lower limit on the error bar associated with the measurement of $\lambda_{k}$ (e.g., Kendall \& Stuart 1967). Toutain \& Appourchaux (1994) showed that these error bars are useful estimates in helioseismology.

Figure 8 shows the errors $\sigma_{\sin i}$ and $\sigma_{\Omega}$ derived from equation (32) for a single $l=1$ mode, plotted as a function of $\sin i$ and $\Omega / \Omega_{\odot}$. This calculation is easier to carry out when $\sin i$ is chosen as an independent parameter instead of $i$. A comparison with Figures 6 and 7 reveals that the error bars obtained by inverting the Hessian have the correct magnitude. By construction they are symmetric about the true parameter values and cannot describe the asymmetric distribution of $i^{*}$ displayed by the Monte Carlo simulations 

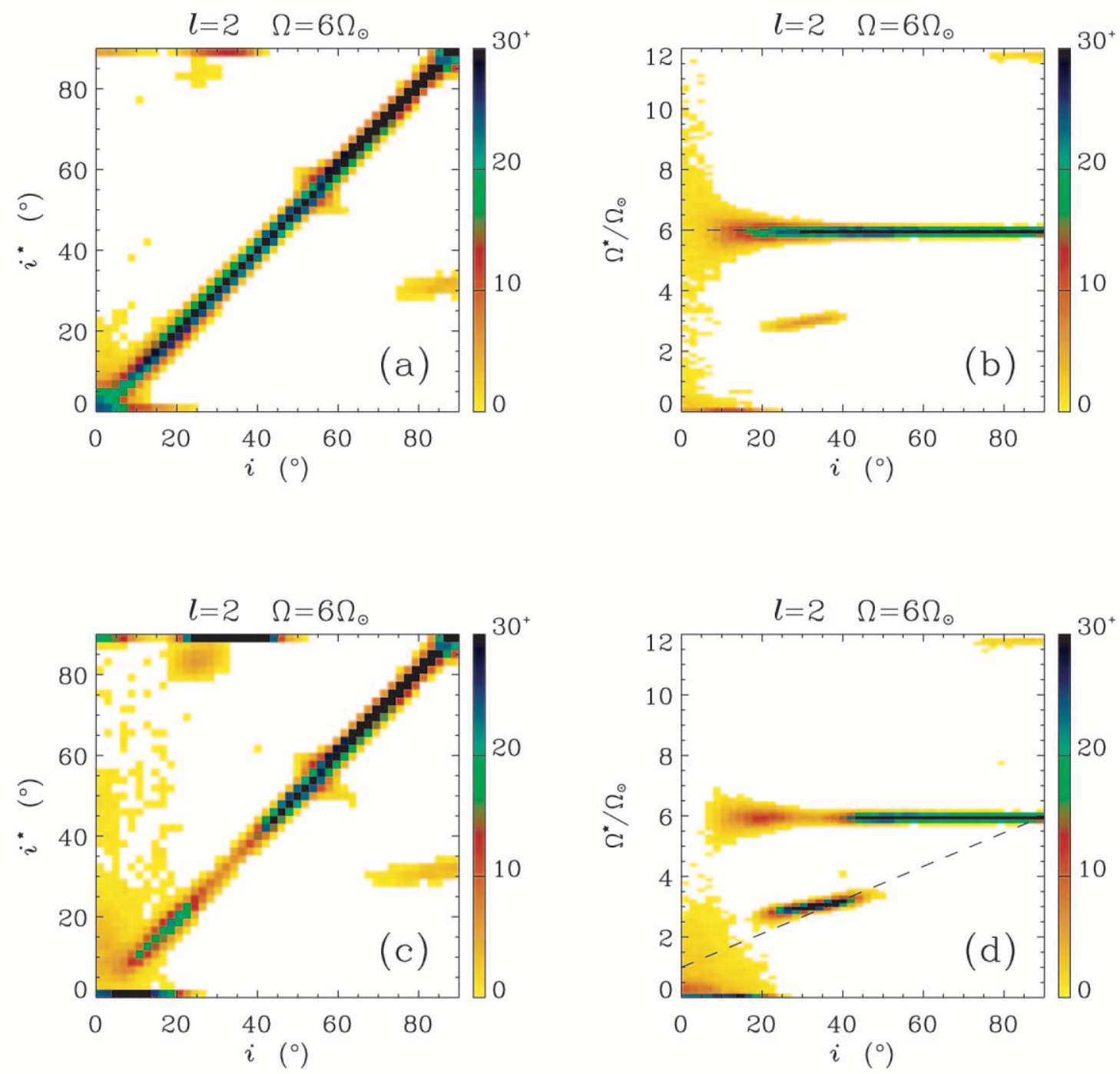

Fig. 5.-Same as Fig. 4, but for an $l=2$ multiplet

(Fig. 7). Of particular interest is the dependence of the error bars on $\Omega$. Figure 8 suggests that it is extremely difficult to determine either $i$ or $\Omega$ for a star with the solar rotation rate when a single mode $l=1$ is taken into consideration.

We have also determined error bars from Monte Carlo simulations for stellar rotation frequencies in the range $1<\Omega / \Omega_{\odot}<10$, although restricted to only $i=30^{\circ}$ and $80^{\circ}$. Medians and error bars are plotted in Figure 9 for a simultaneous fit to three multiplets, $l=0,1,2$. This figure shows that it is realistic to apply asteroseismic techniques for $\Omega \gtrsim 2 \Omega_{\odot}$, with the results being more reliable for $i=80^{\circ}$ than $i=30^{\circ}$. When azimuthal modes are fully resolved (say, $\Omega>3 \Omega_{\odot}$ ), error bars are fairly independent of the rotation rate. Note that for $i=30^{\circ}$ and $\Omega<2 \Omega_{\odot}$, the error bars on $\Omega$ (Fig. $9 b$ ) appear to be decreasing for decreasing $\Omega$. This is an artifact: we simply do not have enough realizations to describe the broad distribution of $\Omega^{*}$ in this range. Furthermore, likelihood estimates $i^{*}$ and $\Omega^{*}$ appear to be biased when $\Omega \lesssim 2 \Omega_{\odot}$. This is likely to be due to our definition of the median (we do not take into account the periodic nature of the distributions).

\section{DISCUSSION}

We have shown in this paper that the inclination angle, $i$, of the axis of rotation of a Sun-like star can in principle be determined with great precision using the techniques of asteroseismology. The observational requirement is a long, nearly continuous time series sampled at a high cadence. The missions COROT and Eddington will provide such data for a large number of stars, with continuous observation for up to 5 and 36 months, respectively. The Danish project MONS (Kjeldsen \& Bedding 1998) aims at targeting individual stars for up to 2 months with a high duty cycle. We have found that it is difficult to estimate the inclination angle for stars with $i<30^{\circ}$, whereby this limit is lower for more rapidly rotating stars. Note however that, statistically, more than $85 \%$ of the stars have $i>30^{\circ}$.

Gough et al. (1995) applied essentially the same method to estimate the inclination angle of the Sun. Solar $p$-modes were observed continuously in irradiance for 160 days in 1988 by the IPHIR experiment (Phobos mission). Gough et al. (1995) found the ratio between the power in $(l=1$, 

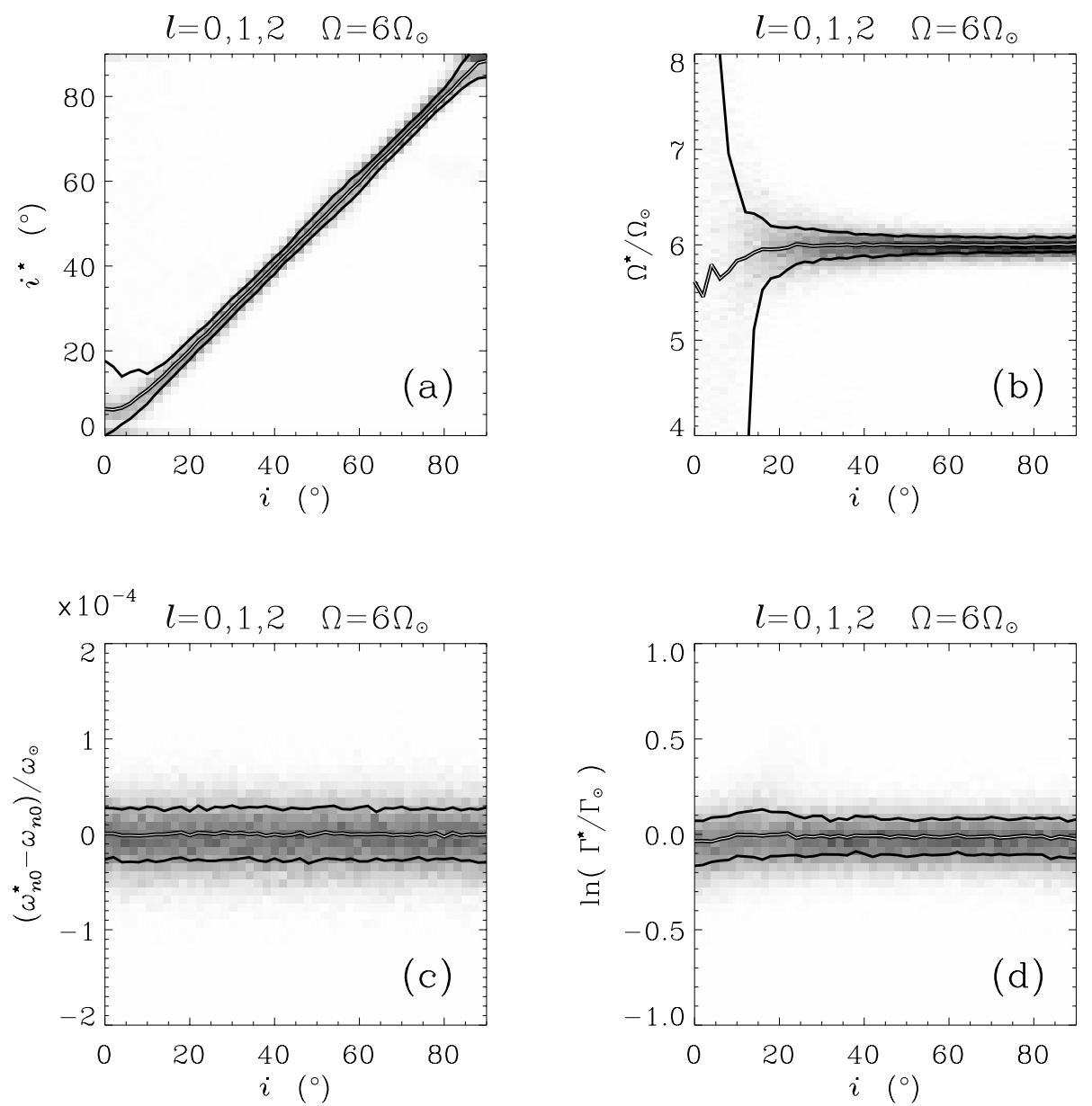

FIG. 6.-Maximum likelihood estimates obtained by fitting three multiplets $l=0,1,2$ simultaneously, for $\Omega=6 \Omega_{\odot}$. The gray scale shows the distributions of $i^{*}, \Omega^{*}$, the FWHM, $\Gamma^{*}$, and the frequency of the $l=0$ mode, $\omega_{n 0}^{*}$, vs. $i$. Plotted are the results of 750 realizations. The thin double lines mark the median of the estimated parameters for each $i$, and $\frac{2}{3}$ of the points fall in between the two thick lines.

$m=0)$ modes and $(l=1, m= \pm 1)$ modes to be less then 0.0009 , i.e., $i>89^{\circ}$.96. They pointed out that at the time of the observations the mean inclination of the rotation axis of the solar surface was $i=85^{\circ}$, corresponding to an expected ratio of 0.015 . Although this discrepancy could be interpreted as a depth dependence in the direction of the solar rotation axis, it is more likely that it is due to a measurement bias. Examination of Figure $4 a$ reveals that the fitting procedure preferentially returns values $i^{*} \approx 90^{\circ}$ for inclination angles in the range $80^{\circ}<i<90^{\circ}$. Besides, the hypothesis of solar rotation about a unique axis is consistent with spatially resolved LOI data (Gizon, Appourchaux, \& Gough 1998). From an astronomer's point of view, it should be clear that Gough et al. (1995) were successful in measuring $i$ with a good precision. We also note that Paunzen, König, \& Dreizler (1998) used a somewhat similar diagnostic, i.e., the amplitude ratio of $m= \pm 1$ and 0 dipole gravity modes, to estimate the inclination of an oscillating pre-white dwarf star.

The precision that can be achieved on the measurement of $i$ depends on each individual star. First of all, acoustic modes must be excited to a sufficiently high amplitude. In this paper we fixed luminosity amplitudes at their solar value with respect to the background noise. Houdek et al. (1999) presented estimates of oscillation amplitudes in main-sequence stars derived from model calculations of stellar convection. They found that velocity amplitudes essentially scale like the mass-to-light ratio of the star, in agreement with an earlier prediction by Kjeldsen \& Bedding (1995). The noise background may also vary. Here we took $S / N=100$, but it has been shown that a useful estimate of $i$ can be obtained when the signal-to-noise ratio is as low as $S / N=20$ (Gizon 2002).

Another condition for determining $i$ is a sufficiently high stellar rotation rate, in order to resolve azimuthal modes split by rotation. This condition is met when the angular velocity of the star, $\Omega$, is at least twice larger than the line width, $\Gamma$, of the modes of oscillation. Since $\Gamma$ is a decreasing function of frequency, the analysis of low-frequency multiplets may be crucial in order to measure the inclination angle of slowly rotating stars. We refer the reader to the work of Houdek et al. (1999) for a study of the variation of mode damping with frequency and stellar parameters. It should be mentioned that the line profile of a peak in the power spectrum may show a small asymmetry. Provided that the asymmetry does not depend on $m$ and that the peaks in a multiplet are resolved, our results will not be affected.

A single realization of the power spectrum will be available for a particular star. How will we know when a measurement of $i$ is meaningful? A large set of Monte Carlo simulations will be essential in assessing the reliability of 

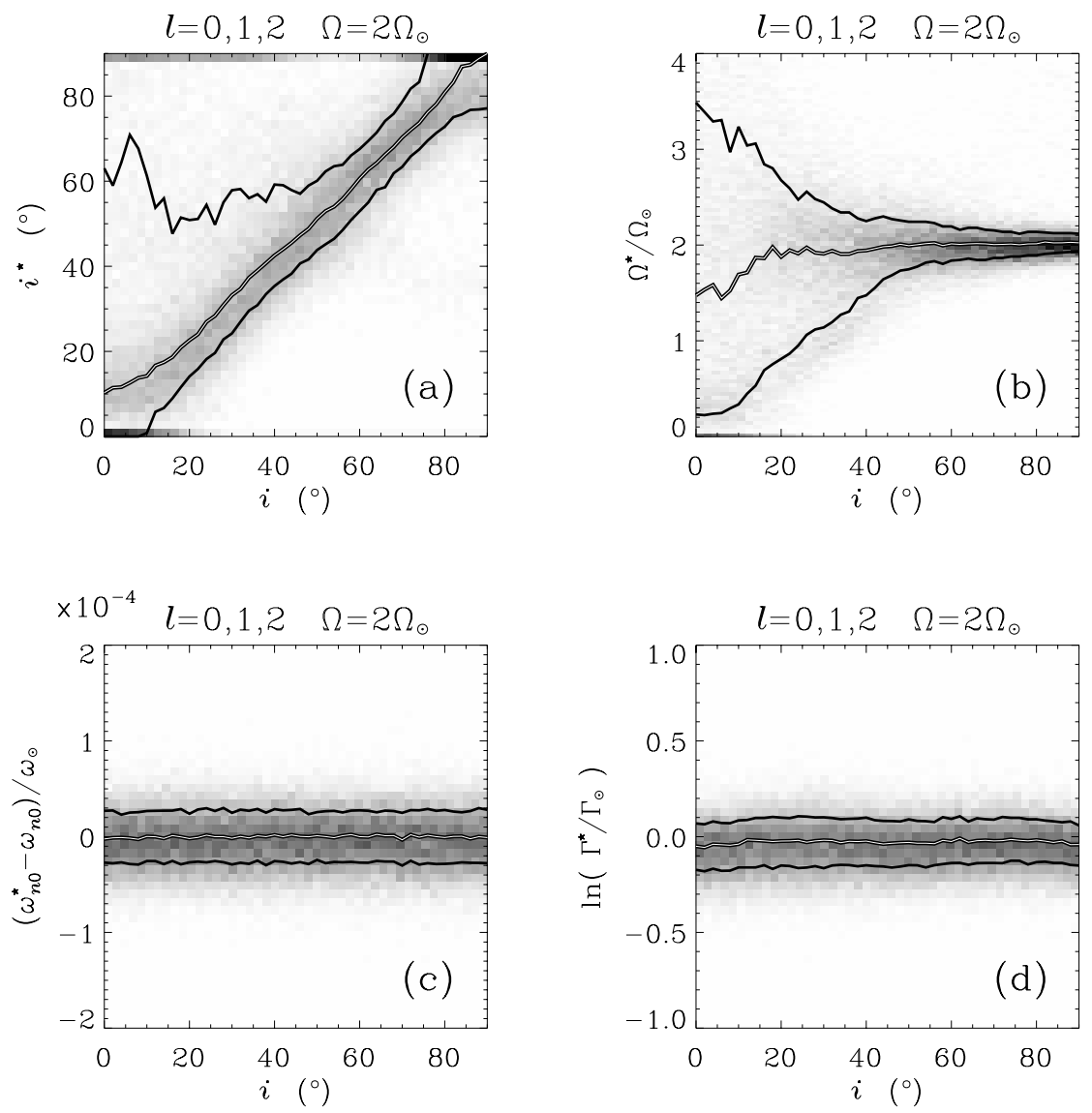

FIG. 7.- Same as Fig. 6, but for $\Omega=2 \Omega$ 。
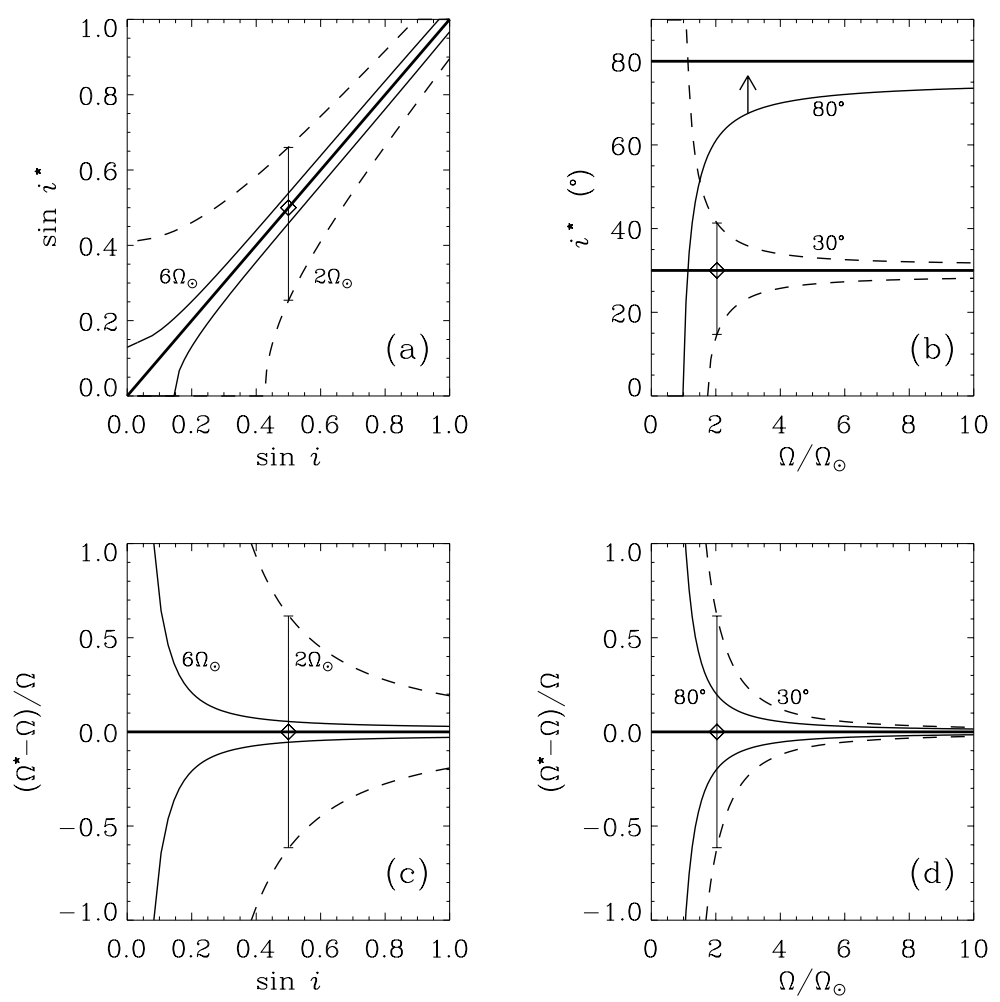

FIG. 8. - Formal error bars obtained by inverting the Hessian for an $l=1$ multiplet. In $(a)$ and $(c)$ the error bars on $i^{*}$ and $\Omega^{*}$ vs. sin $i$ are given for two rotation rates, $\Omega=2 \Omega_{\odot}$ (dashed curves) and $\Omega=6 \Omega_{\odot}$ (solid curves). In $(b)$ and $(d)$ error bars for $i=30^{\circ}$ (dashed curves) and $80^{\circ}$ (solid curves) are plotted vs. $\Omega / \Omega_{\odot}$. A sample error bar for $\Omega=2 \Omega_{\odot}$ and $i=30^{\circ}$ is explicitly plotted. Other parameters are $\Gamma=\Gamma_{\odot}, S / N=100$, and $T=6$ months. 

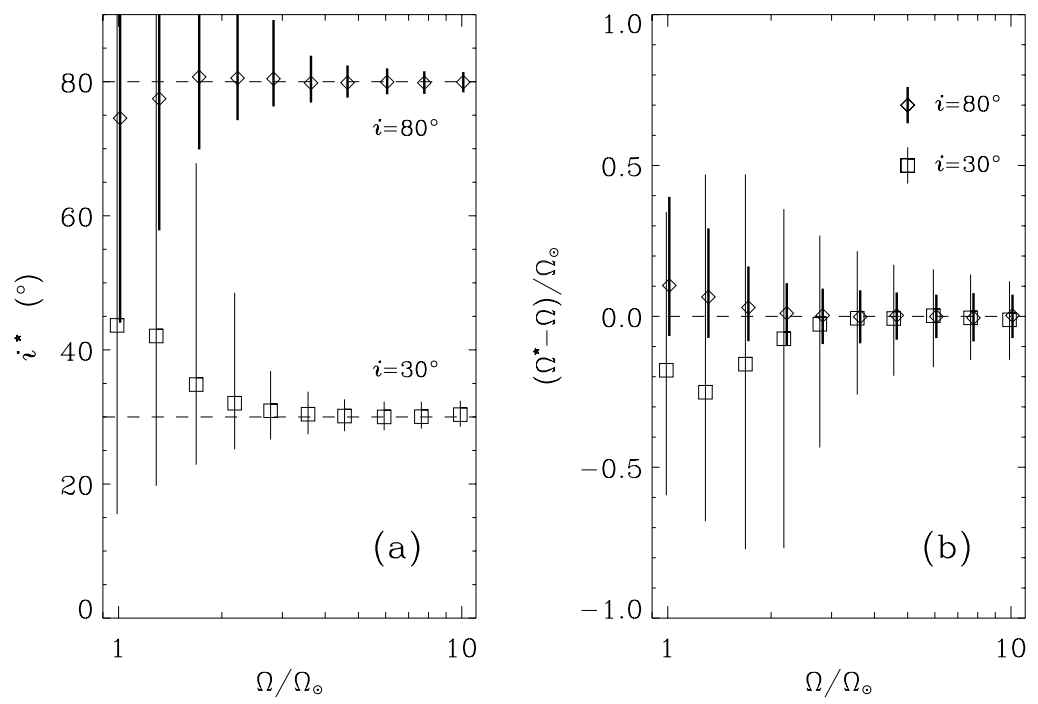

Fig. 9.- Median and error bars for maximum likelihood estimates $i^{*}$ and $\left(\Omega^{*}-\Omega\right) / \Omega_{\odot}$ deduced from Monte Carlo simulations as a function of rotation rate. Results are shown for two input inclinations, $i=30^{\circ}$ (squares and thin error bars) and $80^{\circ}$ (diamonds and thick error bars). Three multiplets $l=0,1,2$ are fitted together. $\Gamma=\Gamma_{\odot}, S / N=100$, and $T=6$ months.

a measurement. As we saw in this study, the answer is likely to be very reliable for several outcomes of the minimization procedure (e.g., large $\Omega^{*}$ and large $i^{*}$ ). In general, the fit also returns an error bar on each estimated parameter that could be used to further increase the confidence in a measurement. Finally, we repeat that the precision on $i$ can be improved by considering more than one multiplet in the power spectrum. The exact number of potentially usable modes depends on the oscillation amplitudes for a particular star. For the Sun, tens of modes can be employed (Fig. 1).

We thank A. C. Birch, J. Schou, and P. H. Scherrer for a critical reading of the manuscript and T. Appourchaux and D. O. Gough for useful discussions. Figure 1 was kindly provided by C. Fröhlich. This work was supported in part by NASA grant NAG 5-8878. L. G. acknowledges the hospitality of the Max-Planck-Institut für Aeronomie.

\section{REFERENCES}

Anderson, E. R., Duvall, T. L., Jr., \& Jefferies, S. M. 1990, ApJ, 364, 699 Appourchaux, T., Chang, H.-Y., Gough, D. O., \& Sekii, T. 2000, MNRAS, 319,365

Appourchaux, T., Gizon, L., \& Rabello-Soares, M.-C. 1998, A\&AS, 132, 107

Baglin, A., et al. (COROT Team). 2001, in Helio- and Asteroseismology at the Dawn of the Millennium, ed. A. Wilson (ESA SP-464; Noordwijk: ESA), 395

Bedding, T. R., et al. 2002, in Asteroseismology across the H-R Diagram, ed. M. Thompson, M. Cunha, \& M. Monteiro (Dordrecht: Kluwer), in press

Bouchy, F., \& Carrier, F. 2001, A\&A, 374, L5

Brown, T. M., Charbonneau, D., Gilliland, R. L., Noyes, R. W., \& Burrows A. 2001, ApJ, 552, 699

Brown, T. M., \& Gilliland, R. L. 1994, ARA\&A, 32, 37

Charbonneau, D., Brown, T. M., Latham, D. W., \& Mayor, M. 2000, ApJ, 529, L45

Dreizler, S., Hauschildt, P. H., Kley, W., Rauch, T., Schuh, S. L., Werner, K., \& Wolff, B. 2003, A\&A, in press

Duvall, T. L., Jr., \& Harvey, J. W. 1986, in Seismology of the Sun and the Distant Stars, ed. D. O. Gough (NATO ASI Ser. C, 169; Dordrecht: Reidel), 105

Dziembowski, W. A. 1977, Acta Astron., 27, 203

Dziembowski, W. A., \& Goode, P. R. 1992, ApJ, 394, 670

Favata, F., Roxburgh, I., \& Christensen-Dalsgaard, J. 2000, Eddington: A Mission to Map Stellar Evolution through Oscillations and to Find Habitable Planets (ESA-SCI (2000)8; Noordwijk: ESA)

Foglizzo, T., et al. 1998, A\&A, 330, 341

Fossat, E. 1981, in Solar Phenomena in Stars and Stellar Systems, ed. R. M. Bonnet \& A. K. Dupree (Dordrecht: Reidel), 75

Fröhlich, C., et al. 1997, Sol. Phys., 170, 1

Gizon, L. 2002, Astron. Nachr., 323, 251

Gizon, L., Appourchaux, T., \& Gough, D. O. 1998, in New Eyes to See inside the Sun and Stars, ed. F.-L. Deubner et al. (Dordrecht: Kluwer), 37

Goode, P. R., \& Thompson, M. J. 1992, ApJ, 395, 307

Gough, D. O., Kosovichev, A. G., \& Toutain, T. 1995, in ASP Conf. Ser. 76, GONG '94: Helio- and Asteroseismology from the Earth and Space, ed. R. K. Ulrich et al. (San Francisco: ASP), 55

Gough, D. O., \& Thompson, M. J. 1990, MNRAS, 242, 25
Hansen, C. J., Cox, J. P., \& Van Horn, H. M. 1977, ApJ, 217, 151

Henry, G. W., Marcy, G. W., Butler, R. P., \& Vogt, S. S. 2000, ApJ, 529, L41

Houdek, G., Balmforth, N. J., Christensen-Dalsgaard, \& Gough, D. O. 1999, ApJ, 351, 582

Kendall, M. G., \& Stuart, A. 1967, The Advanced Theory of Astrophysics: Inference and Relationship, Vol. 2 (2d ed.; London: Butler \& Tanner)

Kjeldsen, H., Arentoft, T., Bedding, T. R., Christensen-Dalsgaard, J., Frandsen, S., \& Thompson, M. J. 1998, in Structure and Dynamics of the Interior of the Sun and Sun-like Stars, ed. S. Korzennik \& A. Wilson (ESA SP-418; Noordwijk: ESA), 385

Kjeldsen, H., \& Bedding, T. R. 1995, A\&A, 293, 87

. 1998, in The First MONS Workshop: Science with a Small Space Telescope, ed. H. Kjeldsen \& T. R. Bedding (Aarhus: Aarhus Univ.), 1

Konacki, M., Torres, G., Jha, S., \& Sasselov, D. 2003, Nature, 421, 507

Ledoux, P. 1951, ApJ, 114, 373

Lissauer, J. J. 1993 , ARA\&A, 31, 129

Marcy, G. W., \& Butler, R. P. 2000, PASP, 112, 137

Mayor, M., \& Queloz, D. 1995, Nature, 378, 355

Messiah, A. 1959, Mécanique Quantique, Tome 2 (Paris: Dunod)

Noyes, R. W., Jha, S., Korzennik, S. G., Krockenberger, M., Nisenson, P., Brown, T. M., Kennelly, E. J., \& Horner, S. D. 1997, ApJ, 483, L111

Paunzen, E. König, M., \& Dreizler, S. 1998, A\&A, 331, 162

Pierce, K. 2000, in Allen's Astrophysical Quantities, ed. A. N. Cox (4th ed.; New York: Springer), 355

Rao, C. R. 1973, Linear Statistical Inference and Its Applications (2d ed.; New York: Wiley)

Safronov, V. S. 1972, Evolution of the Protoplanetary Cloud and Formation of the Earth and the Planets, Israel Program for Scientific Translation, Jerusalem (NASA Tech. Transl. F-677)

Saio, H. 1981, ApJ, 244, 299

Schou, J. 1992, Ph.D. thesis, Aarhus Univ.

Schou, J., \& Buzasi, D. 2001, in Helio- and Asteroseismology at the Dawn of the Millennium, ed. A. Wilson (ESA SP-464; Noordwijk: ESA), 391

Tassoul, M. 1980, ApJS, 43, 469

Toutain, T., \& Appourchaux, T. 1994, A\&A, 289, 649

Toutain, T., \& Gouttebroze, P. 1993, A\&A, 268, 309

Udalski, A., Zebrun, K., Szymanski, M., Kubiak, M., Soszynski, I., Szewczyk, O., Wyrzykowski, L., \& Pietrzynski, G. 2002, Acta Astron., 52,115

Woodard, M. 1984, Ph.D. thesis, Univ. California at San Diego 